\title{
Singularity Extraction for Reflected Sommerfeld Integrals over Multilayered Media
}

\author{
Vladimir V. Petrovic, Senior Member, IEEE, Aleksandra J. Krneta, \\ and Branko M. Kolundzija, Fellow, IEEE
}

\begin{abstract}
A new analytical-numerical method based on singularity extraction is presented for efficient and accurate evaluation of one of the reflected Sommerfeld integrals over multilayered media backed by a conducting (PEC) plane. Method is based on extraction of all the poles of the integrand. The method is demonstrated on one element of the tensor multilayered Green's function, where only the singular part of the integrand, i.e. the reflection coefficient, is taken into consideration, as the cause of the largest calculation difficulties. It was also shown that the method practically eliminates all the branch cut singularities except for one.
\end{abstract}

Keywords - Sommerfeld integrals, singularity extraction, multilayered media

\section{INTRODUCTION}

$\mathrm{C}$ ALCULATION of electromagnetic (EM) fields in the presence of a half-space or multilayered media, whose exact analytical solution is given by the so-called Sommerfeld integrals, basically established by the classical paper [1], is still in the focus of research. Applications are many. Historically the first was radio communication over the earth. Some of the modern applications are ground penetrating radars for detecting buried objects, wall penetrating radars for detecting installations, material defects and inner cracks, through the wall imaging for security applications and geophysical search for gas and oil by using EM waves. However, the most important ones are the increasingly growing application of the multilayered printed circuit boards

Paper received April 6, 2014; accepted October 2, 2014. Date of publication November 15, 2014. The associate editor coordinating the review of this manuscript and approving it for publication was Prof. George Paunovic.

This paper is a revised and expanded version of the paper presented at the 21th Telecommunications Forum TELFOR 2013.

The work presented here is partially supported by Serbian Ministry of Education, Science and Technical development under the grant TR-32005.

V. Petrovic is with TES Electronic Solutions, Zettachring 8, 70567 Stuttgart, Germany (phone: +49-711-7287-7450; e-mail: vladimir.petrovic1@gmx.de).

A. Krneta is with the School of Electrical Engineering, Univ. of Belgrade, Bul. kralja Aleksandra 73, 11120 Belgrade, Serbia (phone: +381-11-3218-353; e-mail: krneta@etf.rs).

B. Kolundzija is with the School of Electrical Engineering, Univ. of Belgrade, Bul. kralja Aleksandra 73, 11120 Belgrade, Serbia (phone: +381-11-3218-329; e-mail: kol@etf.rs).
(PCB) on microwave and millimeter-wave frequencies, where low-frequency approximations and corresponding design methods are not accurate enough. Moreover, such multilayered PCBs often include printed or other antennas placed mostly on the top layer.

A long series of scientific publications on this matter can be traced. A representative list of works can be found, e.g. in [2]. Both exact methods (based on the Sommerfeld integrals) and various approximate methods were developed. The goal of most of these methods is to provide an efficient and accurate evaluation of the Green function for a multilayered structure.

In this paper one of the reflected multilayered Sommerfeld integrals is chosen for demonstration of the method, namely the zz-component of a tensor Green function ( $\overline{\overline{\mathbf{G}}}_{A}$ ) for the magnetic vector-potential [3].

\section{ANALYTICAL Form OF THE MULTILAYERED GREEN FUNCTION $g_{A z z}$}

Consider a multilayered structure, consisting of $M$ layers $(M>1)$, where the bottom layer is a perfectly conducting plane (Fig.1). The structure is considered infinite and uniform in directions perpendicular to $z$-axis. Dielectric layers are non-magnetic and could be lossy or lossless, represented by their equivalent complex permittivity, $\varepsilon_{\mathrm{r} m}$, and thickness, $h_{m}, m=1, \ldots, M-1$. The upper half-space does not have to be vacuum, thus, in general, $\varepsilon_{\mathrm{r} 0} \neq 1$. A source and field point are above the structure and their mutual position is denoted by heights $z_{\mathrm{s}}$ and $z_{\mathrm{f}}$ and the radial distance $\rho$, where any of these three relative coordinates can be zero or approach zero in the limiting case. If all the three coordinates approach zero the tensor Green function becomes singular (tends to infinity).

The element of a tensor Green function chosen for demonstration of the method is $g_{A z z}$, whose compact (normalized) form [3] is given by the Sommerfeld integral

$$
g_{A z z}=\int_{0}^{\infty} F(\alpha, Z, \mathrm{P}) R_{A z z}(\alpha) d \alpha,
$$

where $Z=\beta_{0}\left(z_{\mathrm{s}}+z_{\mathrm{f}}\right), \quad \mathrm{P}=\beta_{0} \rho \quad$ and $\quad \beta_{0}=\omega \sqrt{\varepsilon_{0} \mu_{0}}$. Function $F(\alpha, Z, \mathrm{P})$ comprises the Bessel function and an exponential function and its only singularity (in the complex $\alpha$-plane), as it contains the expression 
$u_{0}=\sqrt{\alpha^{2}-1}$, is the branch point at $\alpha=1$. Function $R_{A z z}(\alpha)$ is often called a reflection coefficient, as it can be interpreted as a reflection coefficient for the plane wave incident to the multilayered media.

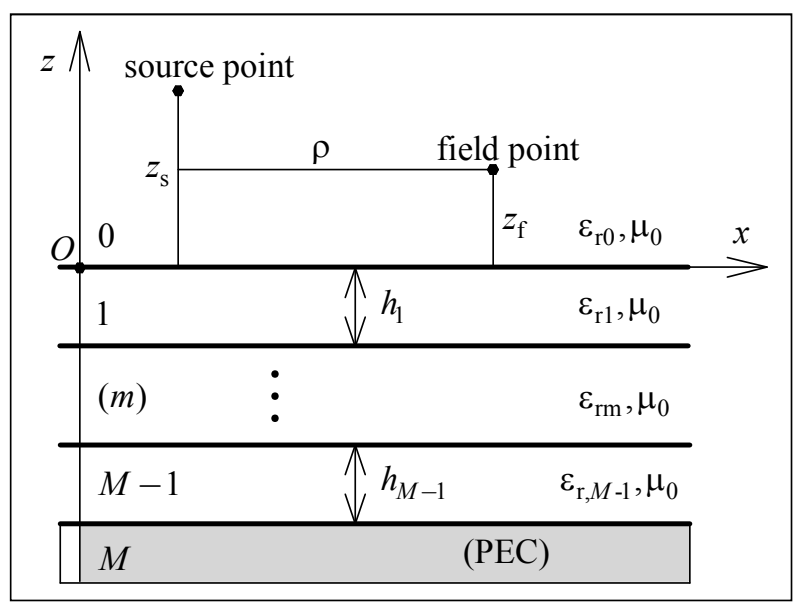

Fig.1. Layered media backed by the PEC plane.

For $\quad \lim _{\alpha \rightarrow \infty} R_{A z z}(\alpha)=R_{A z z \infty} \neq 0 \quad$ significant improvement of integral convergence is obtained when $R_{A z z}(\alpha)$ is represented as $R_{A z z}(\alpha)=R_{A z z \infty}+R_{1}(\alpha)$ and the first of the resulting integrals solved analytically [4], $\int_{0}^{\infty} F(\alpha, Z, \mathrm{P}) R_{A z z \infty} d \alpha=R_{A z z \infty} \mathrm{e}^{-\mathrm{j} R} / R, \quad R=\sqrt{\mathrm{P}^{2}+Z^{2}}$. We will call function $R_{1}(\alpha)$ "a reduced reflection coefficient". Extraction of this term is important as the function $R_{1}(\alpha)$ for large arguments behaves as $\alpha^{-2}$ [3] and alone provides the convergence of the remaining integral, $\int_{0}^{\infty} F(\alpha, Z, \mathrm{P}) R_{1}(\alpha) d \alpha$. This integral cannot be solved analytically. More asymptotic terms could be extracted and the corresponding integrals solved analytically [3], [5]. This will be addressed shortly later in the text.

The oscillating nature of function $F$ and its slow decay to zero for small or zero values of $Z$ make its numerical integration difficult. These two difficulties can, however, be solved in relatively standard and reported ways. A more serious difficulty is that $R_{1}(\alpha)$ contains singularities in the form of poles and branch cut singularities (at points where the branch cuts of the multivalued square root function starts). Every layer introduces one pair of these two types of singularities. For lossless layers these singularities are on the $\alpha$-axis. For layers with small losses (good dielectrics) they are very close to the $\alpha$-axis, what is proven to be an even bigger difficulty for integration [4]. In this paper, the second, more demanding, case will be chosen for demonstration of the method.

In the most demanding case of the Green function evaluation, and that is the case when all the three coordinates, $z_{\mathrm{s}}, z_{\mathrm{f}}$ and $\rho$, approach zero, and, thus both $Z$ and $\mathrm{P}$ in (1) also approach zero, the $F$ function is for small arguments close to one and very slowly decaying. Thus, the integral,

$$
I_{2}=\int_{0}^{\infty} R_{1}(\alpha) d \alpha,
$$

will be considered for demonstration of the proposed method. Due to $\alpha^{-2}$ behavior of $R_{1}(\alpha)$ this integral is convergent. For solving the initial integral (1), the method that will be presented can be combined with techniques for efficient solving the integral if only function $F$ is considered, leading to a hybrid and adaptive overall method, similar to that reported in [3], [4]. Function $R_{A z z}(\alpha)$ is given by the recurrence formula that is presented here according to [6] and after some elementary transformations,

$$
\begin{gathered}
R_{A z z}=\frac{k_{0}-z_{1}}{k_{0}+z_{1}}, \quad z_{m}=k_{m} \frac{z_{m+1}+k_{m} \operatorname{th}\left(u_{m} H_{m}\right)}{k_{m}+z_{m+1} \operatorname{th}\left(u_{m} H_{m}\right)}, \\
m=1, \ldots, M-1, \\
z_{M}=k_{M}, \quad k_{m}=\frac{u_{m}}{\varepsilon_{\mathrm{r} m}}, \quad u_{m}=\sqrt{\alpha^{2}-\varepsilon_{\mathrm{r} m}}, \\
m=0, \ldots, M .
\end{gathered}
$$

Here th( $\bullet)$ is a hyperbolic tangent and $H_{m}=\beta_{0} h_{m}$. In the case that the bottom layer is a PEC plane, is we considered in this work, $k_{M}=0$. In what follows we will consider also complex values of $\alpha$ and will denote such a complex variable as $z$. From the recurrence equations it can be seen that: 1. $R_{A z z}$ is a function of $\alpha^{2}$, thus, any of its series expansions contains only even powers of $\alpha, 2$. $R_{A z z}(z)$ in the complex plane has branch cut singularities at points $\sqrt{\varepsilon_{\mathrm{r} m}}, m=0, \ldots, M-1$, where the function is finite, but its derivative is infinite. It is also shown (e.g. in [7], [8]) that $R_{A z z}(z)$ has a number of poles in the second quadrant $(-\pi / 2<\arg \{z\} \leq 0)$. We will assume that locations of those poles, $z_{0 i}, i=1, \ldots, N$, and their residuums can be efficiently numerically determined (e.g. as explained in [7]).

To derive $R_{1}(\alpha)$ we need to obtain $R_{A z z \infty}=\lim _{\alpha \rightarrow \infty} R_{A z z}(\alpha)$. For this reason we first obtain $\lim _{\alpha \rightarrow \infty} \operatorname{th}\left(u_{m} H_{m}\right)=\lim _{u_{m} \rightarrow \infty} \operatorname{th}\left(u_{m} H_{m}\right)=1$. Next, for $\alpha \rightarrow \infty, z_{m}=k_{m}$ for every $m$ and the result is

$$
R_{A z z \infty}=\lim _{\alpha \rightarrow \infty} \frac{k_{0}-k_{1}}{k_{0}+k_{1}}=\frac{\varepsilon_{\mathrm{r} 1}-\varepsilon_{\mathrm{r} 0}}{\varepsilon_{\mathrm{r} 1}+\varepsilon_{\mathrm{r} 0}} .
$$

This is exactly the same value as for the case when there is only one layer (the first) filling the whole lower halfspace. The value of $R_{A z z \infty}$ is the constant term in the series expansion of $R_{A z z}(\alpha)$ around infinity (which is equivalent to expansion of $R_{A z z}(1 / x)$ around $\left.x=0\right)$. As every expansion of $R_{A z z}(\alpha)$ has only even powers of $\alpha$, the first term in expansion of $R_{1}(\alpha)$ around infinity is the $\alpha^{-2}$ term, as already mentioned. 


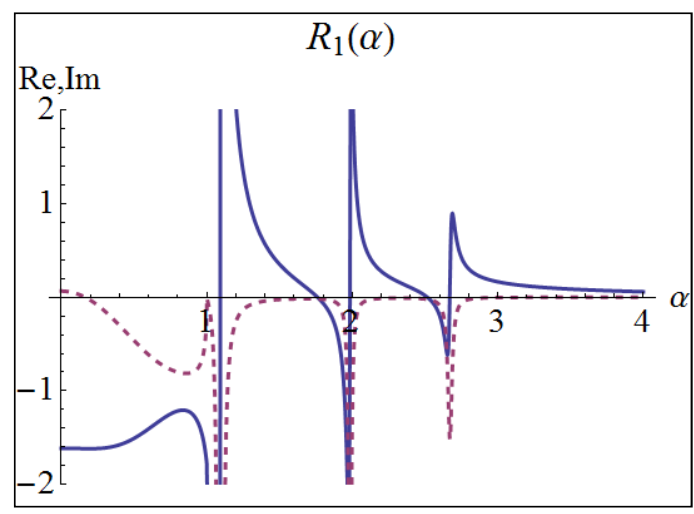

Fig.2. Reduced reflection coefficient along the real axis.

\section{EXTRACTION Of POLES OF THE INTEGRAND}

As an example for demonstration of the method, in the rest of the paper, two-dielectric layered media backed by a PEC plane $(M=3)$, with parameters $\varepsilon_{\mathrm{r} 0}=1-\mathrm{j} 0$ (air), $\varepsilon_{\mathrm{r} 1}=9-\mathrm{j} 0.1, \varepsilon_{\mathrm{r} 2}=4-\mathrm{j} 0.01, H_{1}=2, H_{2}=1$ will be considered.

Graph of the reduced reflection coefficient $R_{1}(\alpha)$, for the chosen parameters of the layered media is shown in Fig. 2, and in the complex plane, denoted $R_{1}(z)$, in Fig. 3 . Large values of $R_{1}(\alpha)$ in Fig.1 are not infinite, because the three poles are somewhat displaced from the real axis ( $\alpha$-axis). However, direct numerical integration of such a function, without the use of special methods is highly inefficient.

In the complex plane, function $R_{1}(\alpha)$ has also branch cuts, one for each of the three functions $u_{m}$, equation (4), namely $u_{0,1,2}$. Each branch cut doubles the multiplicity of the complex functions (as the square root is a two-valued function). These branch cuts can be seen in Fig.4. The multi-valued nature of $R_{1}(\alpha)$ is removed by the condition $-\pi / 2<\arg \{\sqrt{z}\} \leq+\pi / 2$, but branch cut singularities (essential singularities) remain.

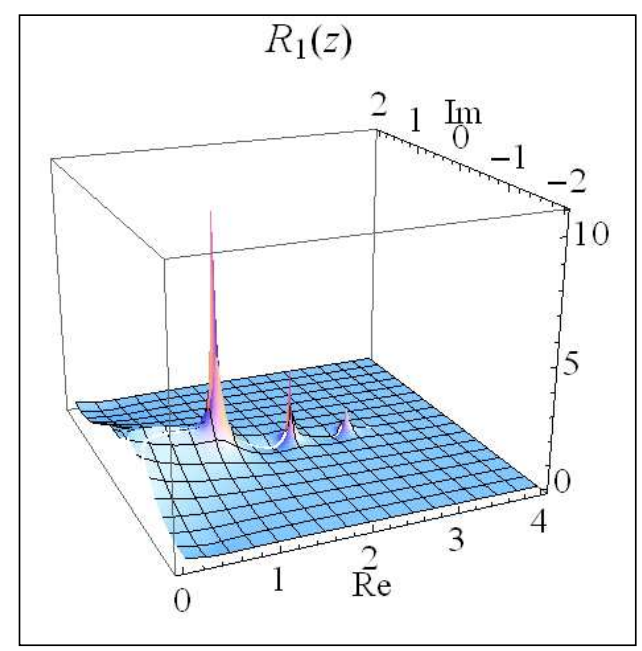

Fig.3. Absolute value of the reduced reflection coefficient in the complex plane.

The method of extracting poles from the integrand is the following. In the complex plane, function $R_{1}(z)$ should be represented as

$$
R_{1}(z)=\sum_{i=1}^{3} R_{\mathrm{s} i} f\left(z-z_{0 i}\right)+f_{3}(z),
$$

where $z_{0 i}$ are locations of poles, the pole singularities are completely included in a properly chosen function $f$ and $R_{\mathrm{s} i}$ are coefficients, directly connected to residuums of $R_{1}(z)$. The remaining function $f_{3}(z)$ is then considered for numerical integration, possibly also after some transformations. Function $f$ must provide that $\int_{0}^{\infty} f\left(z-z_{0 i}\right) d z$ is convergent. The simplest choice for function $f$ seems to be function $\left(z-z_{0 i}\right)^{-1}$. However, this does not lead to the convergence of the integral, as this function behaves in infinity as $\alpha^{-1}$. Instead, we considered the function

$$
f(x)=\frac{\mathrm{e}^{-x^{2}}}{x},
$$

whose primitive function is an exponential integral function, $\operatorname{Ei}(x)$. Further properties of $f(x)$ important here are: 1 . its residuum in the pole $x_{0}=0$ equals one, 2 . its series expansion around infinity is identically equal to 0 , and 3. for real arguments it is an odd function. Due to the first property, $R_{\mathrm{s} i}$ is the residuum of $R_{1}(z)$.

Due to the second property, series expansion of $f_{3}(z)$ at infinity is the same as of the function $R_{1}(z)$. This is a very convenient property. Namely, the majority of techniques for speeding up the integral convergence performs the expansion of the integrand at infinity. By applying function (7), our method enables application of those techniques in an unchanged form.

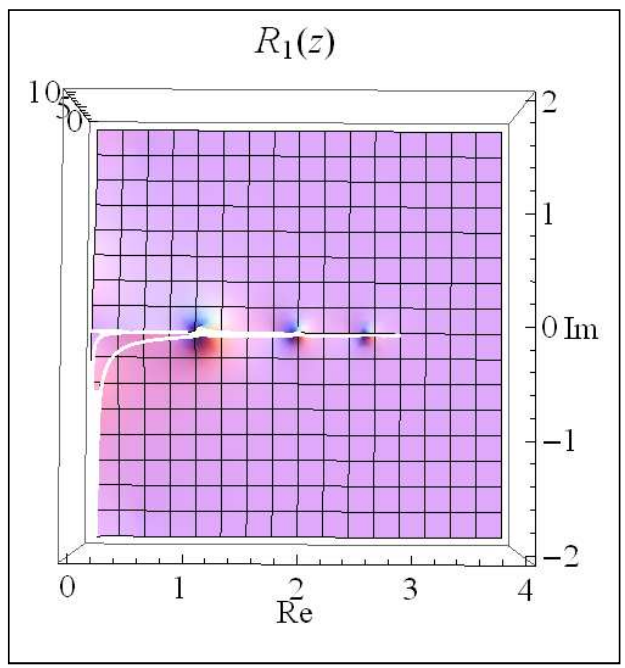

Fig.4. Branch cuts of the reduced reflection coefficient.

Function $f_{3}(z)$ has no poles and is depicted graphically, along the real, $\alpha$-axis in Fig.5. Although with no poles it is obvious that it is not yet suited for numerical integration due to its shape at $\alpha=1$ (branch cut singularity). 


\section{EXTRACTION OF BRANCH CUT SINGULARITIES OF THE INTEGRAND}

The remaining integrand, $f_{3}(z)$ has, theoretically, branch cut singularities at points $\sqrt{\varepsilon_{\mathrm{r} i}}, i=0,1,2$, the first being at $\alpha_{0}=1$ and the other two very close to $\alpha_{2}=3$ and $\alpha_{3}=2$. From Fig. 5 and also after the very detailed analysis around points $\alpha_{2}$ and $\alpha_{3}$, we did not notice any significant singular behavior of the function. Elimination of those two branch cut singularities could be attributed to the fact that the already extracted poles were located very close to those branch cut singularities and that our extraction of those poles practically eliminated those singularities to. This property, which we observed also for other arrangements and parameters of the layered media as in Fig.1, is not yet clearly reported or stated in the published work. This means that only the essential singularity at $\alpha_{0}=1$ needs to be extracted. This is efficiently and completely done by the well-known change of variables [9], $\alpha=\sin \theta$ for $0<\alpha<1$ and $\alpha=\operatorname{ch}(t)$ for $\alpha>1$. The transformed function $f_{3}$ is depicted in Figs. 6 and 7. The size of the interval in which this change of variables is applied is not critical. We adopted, somewhat arbitrarily, $\alpha<2$.

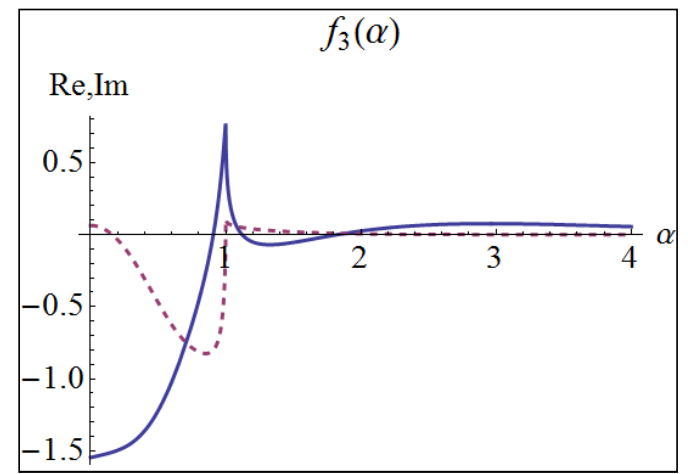

Fig.5. Reflection coefficient after extraction of poles (function $f_{3}$ ), along the real axis.

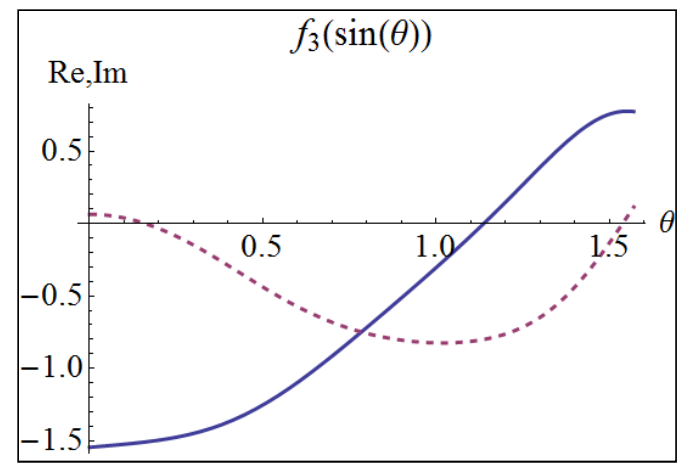

Fig.6. Function $f_{3}$ after the change of variables, for $\alpha<1$.

\section{NUMERICAL RESULTS}

As a numerical demonstration of the proposed method, the method is applied for integration in the interval $(0,4)$, in whose radius around a complex origin all the initial singularities were located. Integration is performed on subintervals of length $\Delta \alpha=1$, by the use of different orders of the standard Gauss polynomial integration formula. Fig. 8 shows the relative error of integration for: 1. direct integration of the reduced reflection coefficient, $R_{1}(\alpha)$, 2. direct integration after extracting the poles, i.e., direct integration of $f_{3}(\alpha)$ without the change of variables around $\alpha=1$ and 3 . as in point 2 , only after a change of variables around $\alpha=1$. Convergence for the direct integration of $R_{1}(\alpha)$ is relatively slow. For the first accurate digit, a formula of the order about 30 must be applied. The fastest convergence, up to errors of about $10^{-5}$ has been achieved by the third method, resulting in error of less than $10^{-5}$ for the formula of the 5 th order. The convergence of the second method is in between.

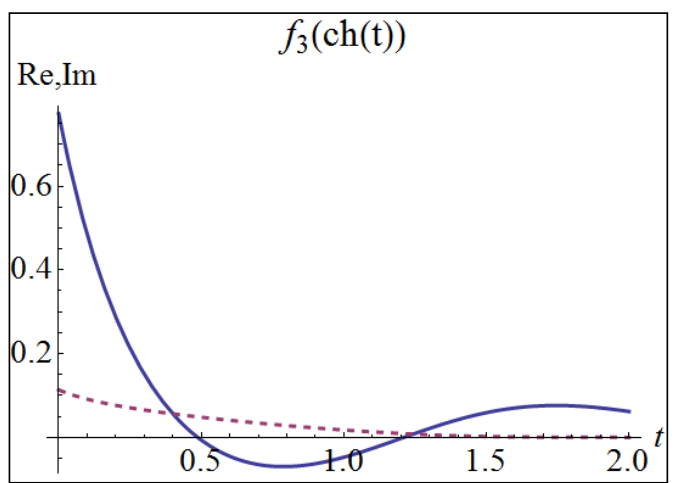

Fig.7. Function $f_{3}$ after the change of variables, for $\alpha<1$.

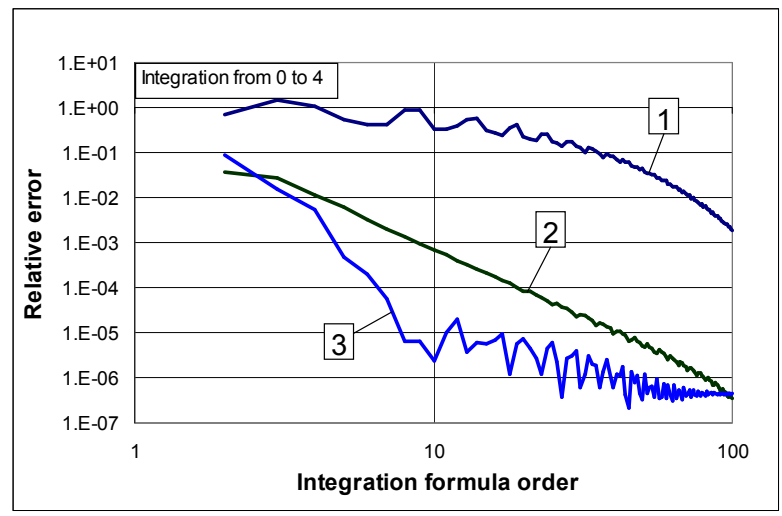

Fig.8. Relative error of integration.

\section{CONCLUSION}

The proposed method for extraction of singularities from the reflection coefficient of the Sommerfeld integrals for the multilayered media efficiently extracts the complex poles of the reflection coefficient. As a side effect of the method, branch cut singularities are also practically removed, except for the singularity at point $(1,0)$ in the complex plane, which is further removed by a simple transformation. This enables efficient numerical integration of the remaining integrand. The method does not change the behavior of the reflection coefficient function for large arguments, i.e. does not alter its series expansion around infinity, which could be very useful for further application of methods for speeding up the convergence of the integral. 


\section{REFERENCES}

[1] A. Sommerfeld A. "Über die Ausbreitung der Wellen in der Drachtlosen Telegraphie," Ann Phys Leipzig 1909; 28 pp.665-736.

[2] K. Michalski "Multilayered media Green's functions in integral equation formulations," IEEE Trans AP 1997, Vol. 45, pp. 508-19.

[3] V.V. Petrovic, A.R. Djordjevic, "General singularity extraction technique for reflected Sommerfeld integrals," AEU - International Journal of Electronics and Communications, Vol.61, Sept 2007, No.8 pp.504-508.

[4] A.R. Djordjevic, M.B. Bazdar, V.V Petrovic, D.I. Olcan, T.K. Sarkar, R.F. Harrington, Awas for Windows, ver. 2.0. Analysis of wire antennas and scatterers, user's manual, Boston MA: Artech House; 2002.
[5] V.V. Petrovic, A.J.Krneta, B.M.Kolundzija, "Method for Improving Convergence of the Reflected Sommerfeld Integrals for a Multilayered Media," Proc. of Telfor Conference 2013,...

[6] J. Wait, Electromagnetic waves in Stratified Media, Oxford University Press, 1996.

[7] A. Alvarez, Applications of the integral equation technique to the analysis and synthesis of multilayered printed shielded microwave circuits and cavity backed antennas, PhD Thesis No.1901, EPFL Lausanne 1998.

[8] D. Djurdjevic, B.D. Popovic, "Entire-domain analysis of thin-wire antennas in layered media," IEE Proc.-Microw. Antennas Propag., Vol 143, No 4, 1996. August.

[9] B.D. Popovic, M.B. Dragovic, A.R. Djordjevic Analysis and synthesis of wire antennas and scatterers, Chichester, UK, Research Studies Press (Wiley), 1982. 